\title{
Shell Injury of the Larynx, Removal Through External Cervical Approach: An Iraqi Experience
}

\author{
Hazem Mohammed Abdel Tawab ${ }^{1,2}$, Khalid Dahham Hamad Luhaib ${ }^{2,3}$ \\ Department of Otorhinolaryngology, Head and Neck Surgery, ${ }^{1}$ Faculty of Medicine, Cairo University, Egypt; ${ }^{2}$ Sultan Qaboos Hospital, \\ Salalah, Oman; ${ }^{3}$ Aljumhoury Teaching Hospital, Mosul, Iraq.
}

\section{Corresponding Author:}

Dr. Hazem M. Abdel Tawab

Email: hazemabdeltawwab77@yahoo.com

This is an Open Access article distributed under the terms of the Creative Commons Attribution License (creativecommons.org/ licenses/by/3.0).

Received Accepted Published

December 8, 2018

March 14, 2019

April 5, 2019

\begin{abstract}
Background: Penetrating neck injuries with laryngeal trauma is uncommon event which can lead to its damage and might cause mortality as well. The incidence of this type of laryngeal trauma increases in war times and areas of conflicts. Different modes of management are recommended depending on the presentation, site and nature of the injury. Case Report: We present this case of a 35 year old male who was exposed to shell injury of the larynx, documented by plain x-ray and CT scan of the neck; and managed by open exploration where midline thyrotomy with removal of the shell were done. Patient had a good recovery and an uneventful follow up period. Conclusion: Causes and incidence of penetrating laryngeal trauma differ between peace and war times. Early recognition and management is required to establish sufficient airway and to secure the patient's life if endangered. Management differs largely depending on the patient's presentation, site of trauma and the nature of the traumatic agent and its velocity.
\end{abstract}

Keywords: Larynx, Neck Injuries, Penetrating Wounds, Radiography, X-Rays.

\section{Introduction}

Penetrating external trauma of the larynx is a rare event [1]. This type of trauma increases during wartime [2]. Due to the great chances of endangering life because of this type of trauma, timely, accurate diagnosis and proper management are essential to preserve the patient's life, and obtain good results [3]. In this paper, we report a case of a 35 year old male with shell injury after a terrorist mortar bomb explosion causing change of voice and removed by external approach through midline thyrotomy.

\section{Case Report}

A 35 year old previously well male was the victim of a terrorist mortar bomb explosion. He presented to the casualty unit at our hospital with multiple bleeding shrapnel wounds involving the right eye, neck, left arm and back. On primary assessment he was conscious and oriented. There was no dyspnea and he was hemodynamically stable. He had obvious hoarseness of voice, but no stridor, dysphagia, hemoptysis, or hematemesis.

Neck examination showed a $2 \times 1 \mathrm{~cm}$ lacerated wound in the right zone II near to midline of the neck with palpable click of the right thyroid cartilage lamina. There were no signs of surgical emphysema and significant bleeding. Vital signs were stable and systemic examinaiton were within normal limits. He had corneal wound in his right eye that was emergently repaired under general anesthesia.

The patient continued to have hoarseness of voice when otolaryngologists were called for assessment. Fiberoptic laryngeal examination revealed normal movements of both vocal cords with no endolaryngeal laceration and no vocal cord hematoma. Plain X-ray revealed a metallic shadow 
in the right side of neck consistent with a shell [Fig.1]. Axial and coronal computed tomography (CT) of the neck further confirmed the presence of shell between two pieces of fractured right thyroid cartilage lamina with minimal fracture displacement [Fig.2,3]. A decision for exploration was made after informed consent and the patient was prepared for surgery. Under general anesthesia, in supine position the neck was explored through a transverse incision and the strap muscles were split in the midline to expose the laryngeal skeleton. Partial laryngofissure was made via midline thyrotomy and the foreign body [Fig.4] was extracted from between the fractured parts of the thyroid cartilage which were approximated and fixed with nylon 3/0 suture and the wound was closed in layers over a drain. Post-operatively, the patient was given systemic antibiotics and antacids for few days. Drain was removed two days after exploration with no further edema or collection. Patient had a smooth post-operative course with no complications. Voice improved dramatically and we received no complaints from the patient regarding speech or feeding. Prior to discharge, fiberoptic laryngoscopy was normal. Patient was seen six weeks and one year after discharge where no abnormality was found by fiberoptic laryngeal examination.

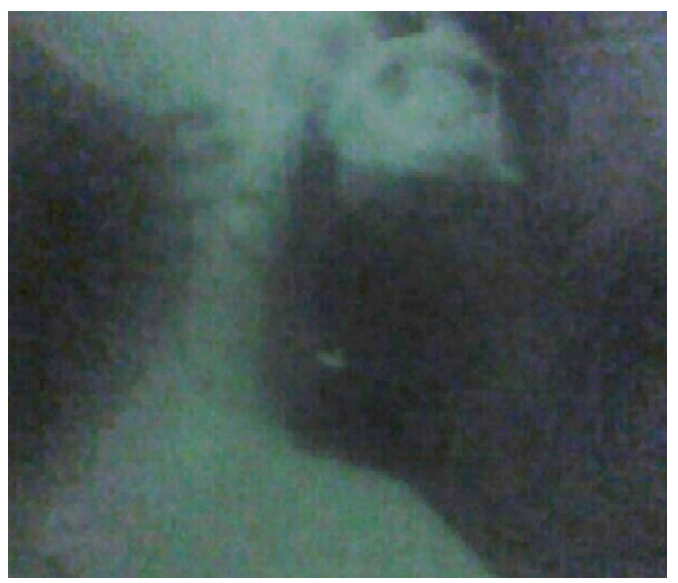

Fig.1: Plain $x$-ray of the neck showing the shell.

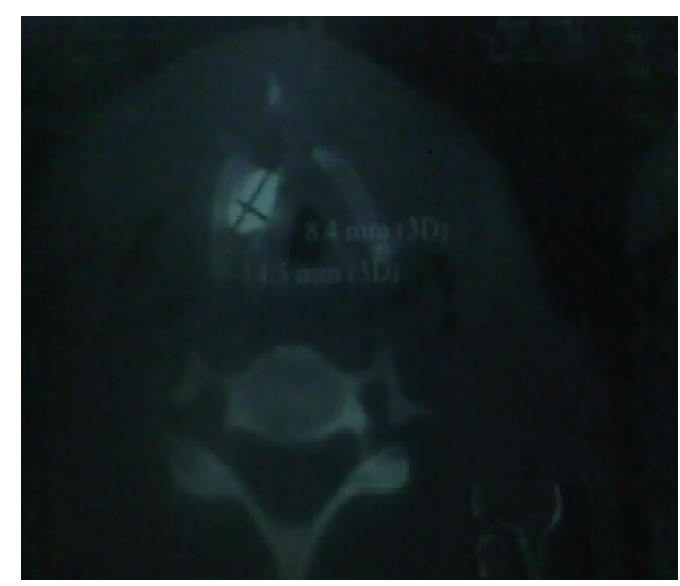

Fig.2: Axial CT scan of the larynx showing the shell.

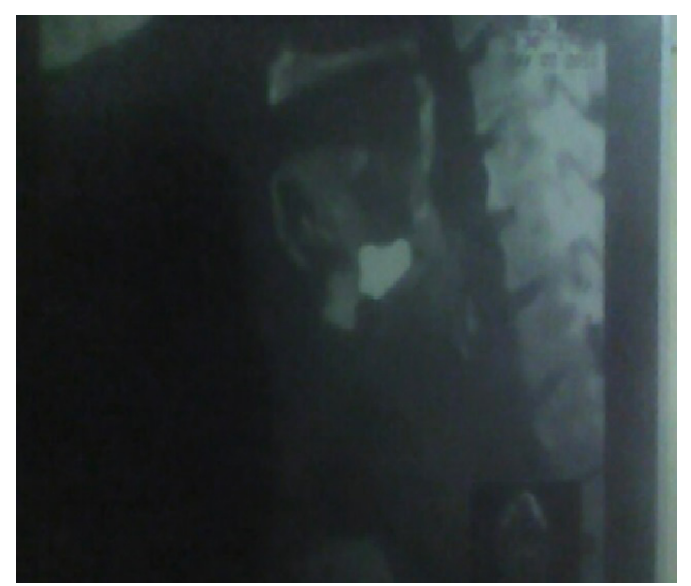

Fig.3: Sagittal section of CT scan showing the shell.

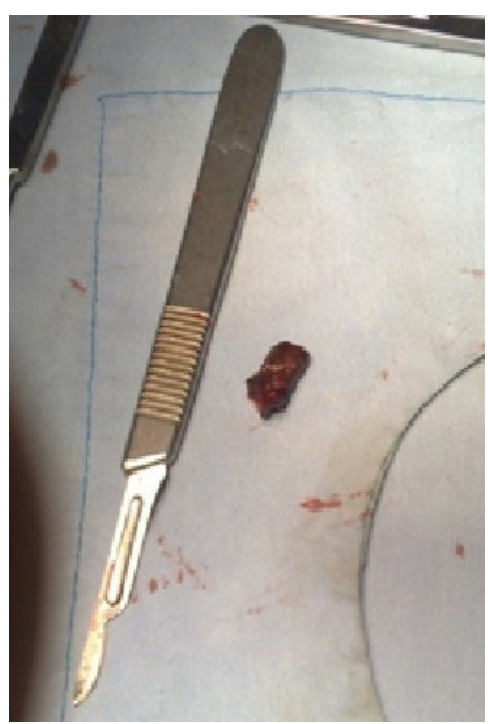

Fig.4: The shell removed from the larynx. 


\section{Discussion}

Trauma to the upper aero-digestive tract can lead to significant morbidity or may have potential lethal results [4]. Gunshot wounds are primarily responsible for penetrating trauma. Injuries vary from minor lacerations to severe disruption of the cartilage, mucosa, soft tissue, nerves, and adjacent structures. The degree of damage is directly related to velocity and mass of the wounding missile [5].

The classic symptoms of laryngeal trauma include hoarseness, laryngeal pain, dyspnea, and dysphagia, however, none of these symptoms correlates well with the severity of injury [6]. When the laryngeal lumen is severely compromised, aphonia and apnea occur, which present the need for immediate establishment of an alternative airway. After trauma, a thorough history taking regarding patient's complaints and physical examination of the neck are needed to identify neurovascular and cervical spinal injuries, active bleeding, expanding hematoma, or other signs of vascular injury. The signs of laryngeal trauma like stridor, hemoptysis, subcutaneous emphysema, and tenderness or deformity of the laryngeal skeleton should be excluded in every single case of neck trauma. Laryngeal examination by fiberoptic laryngoscopy and radiological testing are required to evaluate the extent of laryngeal injury in cases of external laryngeal trauma [3].

In this paper, patient's injury was found to be in right side of the neck zone II with no considerable hematoma or neck emphysema. Some authors advocate mandatory exploration in cases of zone II injuries [3] if serial radiological examination is not feasible. However, with modern sensitive imaging techniques, the term (no zone) had been introduced where stable patients with no airway or vascular injury are better evaluated with CT scans or ultrasound and angiography [7].

CT should be done for patients with suspected laryngeal injury and inconsistent symptoms of injury such as hoarseness as in our patient where only change of voice was present with minimal neck injury. CT in such conditions can be used to diagnose patients with minimally displaced midline or lateral thyroid cartilage fractures that are minimally symptomatic and if left untreated, might lead to long-term phonatory disturbances because of disruption of the laryngeal valve mechanisms [8].

For patients with no fractures, minor lacerations and minimal edema, minimal airway symptoms; management should be observation and supportive medical care [6]. For small nondisplaced fractures, with no airway compromise, conservative management including steroids and repeat laryngoscopy to confirm patent functioning airway is only needed. For any comminuted or large fractures, anterior commisure lesions, exposed cartilage or displaced fractures open reduction will be needed [9].

Overall treatment should be individualized according to presenting complaints. In the reported case, persistent change of voice changed the management from conservative (due to minor injuries) to surgical based on $\mathrm{x}$ ray and $\mathrm{CT}$ findings and his persistent complaint. The most reliable method for securing an airway in a patient with laryngeal injury is to do a tracheotomy while the patient is awake. Endotracheal intubation may further damage the larynx and can interfere with its subsequent examination and repair.

In our patient here, conservative management was the primary method of treatment till his complaint of voice change continued then further investigations were done and decision for exploration was taken. Due to the absence of any mucosal damage or lacerations or exposed cartilage, endotracheal intubation was done successfully. Then, midline thyrotomy was achieved, foreign body shell removed and thyroid fracture repaired. Closure of the partial laryngofissure was then carried out using no absorbable sutures over a drain. 
In patients with non-displaced fractures of the thyroid cartilage, healthy true vocal fold motion, and no injury to the anterior commissure conservative management is usually indicated [10]. Serial flexible fiberoptic laryngoscopic examinations should be performed to ensure proper healing. In patients with more severe injuries, surgical exploration is performed. Controversy exists as to the optimal time for repair. Some authors prefer delay of repair for 3-5 days to allow edema to subside and for easier identification of mucosal lacerations. However, best results have been obtained with early repair, avoiding the morbidity of leaving open wounds in a contaminated field [11].

All patients with laryngeal trauma should continue to receive post-operative antibiotics for 5-7 days to reduce the risk of infection and granulation tissue formation. The head of the bed should be elevated, as tolerated, to minimize edema. Patient should be encouraged to ambulate as soon as bearable. Antacids and $\mathrm{H}_{2}$-blockers should be routinely used to prevent reflux, which may cause increased scarring of laryngeal tissues.

The outcome after laryngeal trauma depends on the extent of the original injury and quality of repair if needed. In patients who do not require surgical intervention, full return of function is excellent [11]. Patients requiring surgical intervention have an excellent chance of adequate to good voice [12]. Long-term complications after repair are uncommon. Our patient had full recovery of his voice with no reported early or late complications during his serial laryngoscopic examination over a period of one year following trauma event.

\section{Conclusion}

Care should be given in cases of neck trauma especially in war times not to miss a case with penetrating injury of the larynx. Proper management of penetrating laryngeal trauma with early diagnosis, proper repair as needed and close follow up gives excellent results with good airway and phonatory functions.

Contributors: HMAT: manuscript editing, patient management; KDHL: manuscript writing, patient management. HMAT will act as guarantor. All authors approved the final version of this manuscript.

Funding: None; Competing interests: None stated.

\section{References}

1. Bolzoni A, Peretti G, Piazza C, Pedruzzi B, Nicolai P. Penetrating foreign body mimicking supraglottic carcinoma. Ann Otol Rhinol Laryngol. 2005;114:577578.

2. Banfield GK, Chowdhury CR, Brookstein R. Penetrating laryngeal injury: two case reports from Bosnia. J Laryngol Otol. 1995;109:144-146.

3. Thompson EC, Porter JM, Fernandez LG. Penetrating laryngeal trauma: an overview of management. J Oral Maxillofac Surg. 2002;60:918-923.

4. Thevasagayam MS, Pracy P. Laryngeal trauma: a systematic approach to management. Trauma. 2005;7:8794.

5. Lucente FE, Mitrani M, Sacks SH, Biller HF. Penetrating injuries of the larynx. Ear Nose Throat J. 1985;64:406.

6. Schaefer SD. Primary management of laryngeal trauma. Ann Otol Rhinol Laryngol. 1982;91:399-402.

7. Shiroff A, Gale SC, Martin ND, Marchalik D, Petrov D, Ahmed HM, et al. Penetrating neck trauma: a review of management strategies and discussion of the 'no zone' approach. Am Surg. 2013;79:23-29.

8. Stanley RB, Cooper DS, Florman SH. Phonatory effects of thyroid cartilage fractures. Ann Otol Rhinol Laryngol. 1987;96:493

9. Jalisi S, Zoccoli M. Management of laryngeal fractures, a 10 year experience. J Voice. 2009;25:473-479.

10. Stanley RB, Cooper DS, Florman SH. Phonatory effects of thyroid cartilage fractures. Ann Otol Rhinol Laryngol. 1987;96:493-496.

11. Kleinsasser NH. External trauma to the larynx classification, diagnosis, therapy. Eur Arch Otorhinolaryngol. 2000;257:439-444.

12. Krekorian EA. Laryngopharyngeal injuries. Laryngoscope. 1975;85:2069-2086. 